\title{
RESÍDUO DE SISAL SOBRE Meloidogyne javanica EM PLANTAS DE ALFACE
}

\author{
Josilda Cavalcante Amorim Damasceno ${ }^{1}$; Ana Cristina Fermino Soares ${ }^{2}$; Anailde \\ Cavalcante dos Santos ${ }^{3}$, Maria Santos Conceição $0^{4}$; Zozilene Nascimento Santos Teles ${ }^{5}$ \\ 'Doutora em Ciências Agrárias- Universidade Federal do Recôncavo da Bahia- UFRB \\ (josildadamasceno@gmail.com), Cruz das Almas-BA \\ ${ }^{2}$ Professora de Pós Graduação em Ciências Agrárias e Microbiologia Agrícola UFRB, \\ Cruz das Almas, BA \\ ${ }^{3}$ Mestrando em Ciências Agrárias, UFRB, Cruz das Almas, BA \\ ${ }^{4}$ Mestrando em Solos e Qualidade de Ecossistemas, UFRB, Cruz das Almas, BA \\ ${ }^{5}$ Técnico Administrativo, UFRB, Cruz das Almas - BA
}

\section{Recebido em: 02/10/2017 - Aprovado em: 21/11/2017 - Publicado em: 05/12/2017 DOI: 10.18677/EnciBio_2017B59}

\begin{abstract}
Este trabalho teve o objetivo de avaliar o efeito do resíduo líquido fresco de sisal no controle de Meloidogyne javanica em alface. Mudas de alface foram transplantadas em casa de vegetação. Foi feita a inoculação sete dias após o transplante. Sete dias após a inoculação, procedeu-se a aplicação do resíduo de sisal. Foram inoculados 1000 juvenis de $M$. javanica e aplicados $100 \mathrm{~mL}$ do resíduo líquido de sisal nas concentrações de 0,0\%( tratamento controle), 5,0\%, 10,0\%, 15,0\%, 20,0\%, 25,0\% e $30,0 \%$. O delineamento foi inteiramente ao acaso com dez repetições. Após 50 dias plantas foram coletadas e analisadas quanto à massa seca da parte aérea e das raízes, o número de galhas e de massas de ovos nas raízes. Houve maior produção de massa seca da parte aérea e das raízes na concentração de $20 \%$, com incrementos de $42,0 \%$ e 43,4\%. Nesta mesma concentração, a redução no número de galhas e de massas de ovos por planta foi de $90,1 \%$ e $76,6 \%$ respectivamente. Concentrações de $25 \%$ e $30 \%$ reduziram o peso da massa seca de parte aérea e das raízes da alface os quais são indicativos de fitotoxicidade. Estudos futuros serão conduzidos visando comprovar a ação nematicida deste resíduo no controle de $M$. javanica em alface a campo.
\end{abstract}

PALAVRAS-CHAVE: Agave sisalana Perrine ex Engelm, controle orgânico, nematoides das galhas.

\section{SISAL RESIDUE ON Meloidogyne javanica ON LETTUCE PLANTS}

\section{ABSTRACT}

The objective was to study the effect of sisal liquid residue in the control of Meloidogyne javanica in lettuce. In greenhouse, lettuce seedlings were transplanted and seven days later, the inoculation was done. Seven days after inoculation, the sisal residue was applied. The plants were inoculated with 1000 juveniles of $M$. javanica and $100 \mathrm{~mL}$ of sisal liquid residue at concentrations of $0.0 \%$ (control treatment), $5.0 \%, 10.0 \%, 15.0 \%$, 
$20.0 \%, 25.0 \%$ and $30.0 \%$. The experiment was done in a completely randomized design with ten replications per treatment. After 50 days, plants were collected and analyzed for dry masses of shoots and roots, number of galls and egg masses in the roots. There was a higher dry weight of shoots and roots in the concentration of $20 \%$, with increases of $42.0 \%$ and $43.4 \%$. At this same concentration, the reduction in the number of galls and egg masses per plant was $90.1 \%$ and $76.6 \%$, respectively. Concentrations of $25.0 \%$ and $30.0 \%$ reduced the weight of dry mass of shoots and roots, being a strong indicative of phytotoxicity. Future studies should be conducted to test the nematicidal effect in lettuce plants under field conditions.

KEYWORDS: Agave sisalana Perrine ex Engelm; organic control; root-knot.

\section{INTRODUÇÃO}

A alface (Lactuca sativa L.) é a mais importante hortaliça folhosa cultivada mundialmente, planta herbácea anual, pertencente à família Asteraceae, constitui o grupo de hortaliças folhosas de maior consumo no Brasi, sendo consumida in natura em forma de salada (SALA ; COSTA, 2012). O Brasil produziu em 2104 cerca de 525.602 toneladas. O Estado de São Paulo é considerado maior produtor nacional, correspondendo a cerca de $32 \%$ da produção brasileira (HORTIBRASIL, 2015). Entretanto, esta cultura enfrenta diversos problemas causados por patógenos, dentre os quais, destacam-se aqueles do gênero Meloidogyne spp., conhecidos como nematoide das galhas.

Nas plantas atacadas por estes nematoides, ocorrem alterações no sistema radicular, devidos a hipertrofia e hiperplasia das células do parênquima vascular da raiz, provocados pela formação de galhas no seu sistema radicular (CORDEIRO et al., 2005).

A presença destes patógenos nos sistema radicular, prejudica a absorção de água e nutrientes do solo, consequentemente, as plantas tornam-se murchas nas horas mais quentes do dia, com coloração amareladas, com tamanho e volume foliar reduzidos, tornando-as inviáveis para comercialização (ORNAT ; SORRIBAS, 2008).

Métodos alternativos, como utilização do controle biologico (JÚNIOR et al., 2017), a incorporação ao solo de compostos orgânicos de origem vegetal, tortas de resíduos, extratos de plantas (MAIA et al., 2015; FREIRE, 2016), podem ser utilizadas no manejo de pragas e doenças ( RIBEIRO et al., 2012; RIBEIRO et al., 2013; GOMES ; LIMA, 2014). A utilização do resíduo do desfibramento das folhas de sisal (Agave sisalana Perrine ex. Engelm) no controle de fitonematoides representa mais uma alternativa para os produtores, além de ter baixo custo e não oferecer riscos de contaminação do ambiente (DAMASCENO et al., 2015). Trabalhos desenvolvidos por Silva et al. (2016) avaliando a reação de genótipos de soja infectados com Pratylenchus bachyurus, incluiram Crotalária spectabilis como padrão de resistência ao patógeno e concluíram que esta planta pode ser adotada no manejo deste nematoide, visto que, o nematoide apresentou um baixo fator de reprodução na mesma.

Júnior et al. (2017) avaliaram o efeito de diversos extratos aquosos e alcoólicos de variedades de pimentas do gênero Capsicum no controle de diversos fungos. Segundo os autores, os extratos demonstraram efeito fungicida. O sisal pertence a família Agavaceae é uma planta monocotiledônea originária do México. O Brasil é o maior produtor e exportador mundial da fibra de sisal e, correspondendo a $50 \%$ desse total, 
sendo o Estado da Bahia responsável por 95,8\% da produção nacional (CONAB, 2015). A fibra de sisal representa somente $4,0 \%$ da folha e os resíduos sólidos e aquosos constituem os $96,0 \%$ restantes, sendo $80,0 \%$ de resíduo líquido (FILHO et al., 2013), que são abandonados nas propriedades rurais, sem qualquer aproveitamento.

Botura et al. (2013) encontraram como os principais constituintes oriundos do metabolismo secundário do resíduo de sisal, alcaloides, compostos fenólicos, cumarinas, saponinas, flavonoides e taninos. Estas substâncias estão relacionadas, principalmente, ao mecanismo de defesa das plantas, e podem apresentar efeito inibitório contra fitonematoides (CHITWOOD, 2002).

Em plantas do gênero Agave foram constatadas ações biocidas com relação à insetos (BARRETO et al., 2010), à nematoides em caprinos e ovinos (DOMINGUES et al., 2010; BOTURA et al., 2013), nematoides em plantas (DAMASCENO et al., 2015; JESUS et al., 2015). Neste contexto, este trabalho objetivou avaliar o efeito do resíduo líquido de sisal no controle de Meloidogyne javanica em plantas de alface.

\section{MATERIAL E MÉTODOS \\ Obtenção e desinfestação de indivíduos de Meloidogyne javanica:}

Para obtenção dos indivíduos, raízes de tomateiro cv. 'Santa Cruz Kada', cultivado em casa de vegetação, inoculadas com $M$. javanica, foram lavadas com água potável e trituradas em liquidificador por 20 segundos com uma solução de hipoclorito de sódio a 0,5\% (BONETI ; FERRAZ, 1981).

\section{Obtenção do resíduo líquido de sisal}

O resíduo líquido fresco foi obtido durante o processo de desfibramento das folhas de sisal, em propriedade rural localizada no município de Valente, BA e transportado, sob refrigeração, para o Laboratório de Microbiologia Agrícola da UFRB, onde foi filtrado por três vezes em peneira de 400 mesh e, mantido em freezer a $-20^{\circ} \mathrm{C}$, até a utilização nos estudos in vitro e in vivo. Os resíduos fresco apresentou pH 5,0 e foi ajustado com $\mathrm{NaOH}(10 \%)$ para 6,5 .

\section{Controle de Meloidogyne javanica na alface com resíduo líquido de sisal}

O ensaio foi conduzido em casa de vegetação, na Universidade Federal do Recôncavo da Bahia, em Cruz das Almas, latitude 12³9'11"S, longitude 3907'19"W e altitude de $212 \mathrm{~m}$, com clima Tropical Quente e Úmido, em solo do tipo Latossolo Amarelo. As mudas de alface cv. Regina foram germinadas em substrato Vivatto Slim ${ }^{\circledR}$, o mesmo é composto por casca de pinus, moinha de carvão vegetal e turfa, com um $\mathrm{pH}$ de 5,6 de acordo com o fabricante, posteriormente, transplantadas aos 20 dias após a germinação, posteriormente, foram transplantadas para vasos plásticos de $2 \mathrm{~L}$, contendo a mistura de solo e areia (2:1), esterilizada em autoclave a $120{ }^{\circ} \mathrm{C}$ por 1,5 horas, em dois dias consecutivos. Aos sete dias após o transplantio, fez-se a inoculação de $1000 \mathrm{~J} 2$ de M. javanica por planta. Quatro dias após a inoculação dos nematoides, $100 \mathrm{~mL}$ do resíduo líquido, nas diferentes concentrações, foram incorporados ao solo em cada um dos vasos e duas aplicações posteriores foram realizadas a cada quinze dias, após a data de aplicação da primeira.

$O$ delineamento foi inteiramente ao acaso constando de sete tratamentos: $0,0 \%$ (controle), 5,0\%, 10,0\%, 15,0\%, 20,0\%, 25,0\% e 30,0\% com dez repetições. A parcela experimental foi constituída por uma planta por vaso. Aos 50 dias após a inoculação, as 
plantas foram coletadas e analisadas a massa seca da parte aérea e das raízes, número de galhas e de massa de ovos por planta e por grama de raiz.

Para a contagem dos números de massas de ovos e de galhas, amostras de raízes frescas foram separadas e coloridas por imersão em solução de Ponceau 4R a $1 \%$, um corante artificial comestível, durante 20 minutos (DAMASCENO et al., 2016). Os dados foram submetidos às análises de variância e de regressão em função das concentrações do resíduo, utilizando o programa estatístico SISVAR.

\section{RESULTADOS E DISCUSSÃO}

Houve diferença significativa entre os tratamentos para a produção de massa seca da parte aérea e massa seca das raízes. Verificou-se maior produção de massa seca da parte aérea e das raízes na concentração de $20,0 \%$ quando comparado com o controle, com incrementos de $42,0 \%$ e $43,4 \%$, respectivamente (Figuras $1 \mathrm{~A}$ e B).

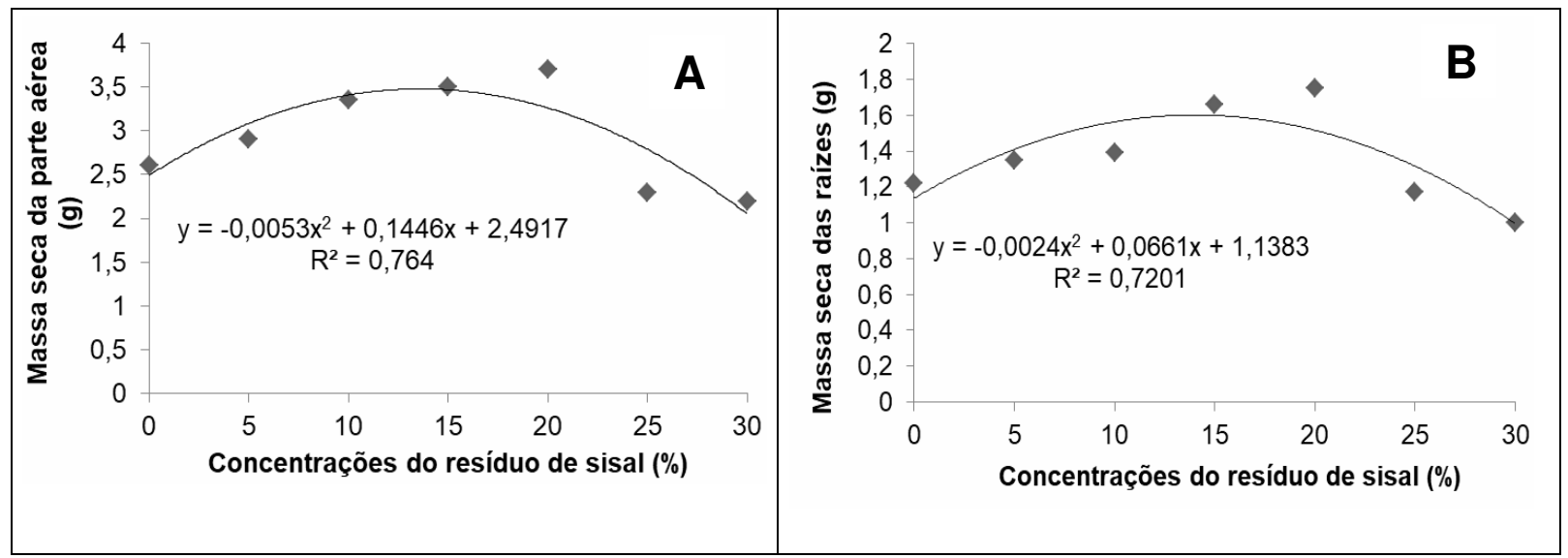

FIGURA 1. A) Massa seca da parte aérea e B) das raízes de alface tratadas com diferentes concentrações de resíduo líquido de sisal.

Entretanto, nos tratamentos com 25,0\% e 30,0\% do resíduo líquido de sisal, houve efeito fitotóxico para as plantas, com redução significativa da massa seca da parte aérea e das raízes da alface (Figuras 1 A e B). A aplicação do resíduo líquido de sisal causou redução dos danos provocados pelos nematoides nas raízes das plantas em todas as concentrações avaliadas, demonstrando efeito nematicida (Figuras 2 e 3 ).

Encontrou-se o modelo linear obtido no ajuste para o efeito das concentrações do resíduo de sisal sobre o número de galhas e de massas de ovos por planta, indicou a tendência de redução deste patógeno com o aumento da concentração do resíduo. Houve redução no número de galhas por planta de 60,3\%, 77,5\%, 81,3\%, 90,1\%, $93,0 \%$ e $93,5 \%$ para as concentrações a $5,0 \%, 10,0 \%, 15,0 \%, 20,0 \%, 25,0 \%$ e $30,0 \%$, respectivamente, quando comparados com o tratamento controle (sem o resíduo - $0 \%$ ) (Figura 2 A).

Em relação às massas de ovos por planta, a redução deste dano nas raízes nas concentrações de $10,0 \%, 15,0 \%, 20,0 \%, 25,0 \%$ e $30,0 \%$, foi de $50,8 \%, 64,6 \%, 76,6 \%$, $82,4 \%$ e 90,2\%, respectivamente, em relação ao controle (sem resíduo) A concentração de $5,0 \%$ não diferiu estatisticamente do tratamento controle (Figura 2 B). 


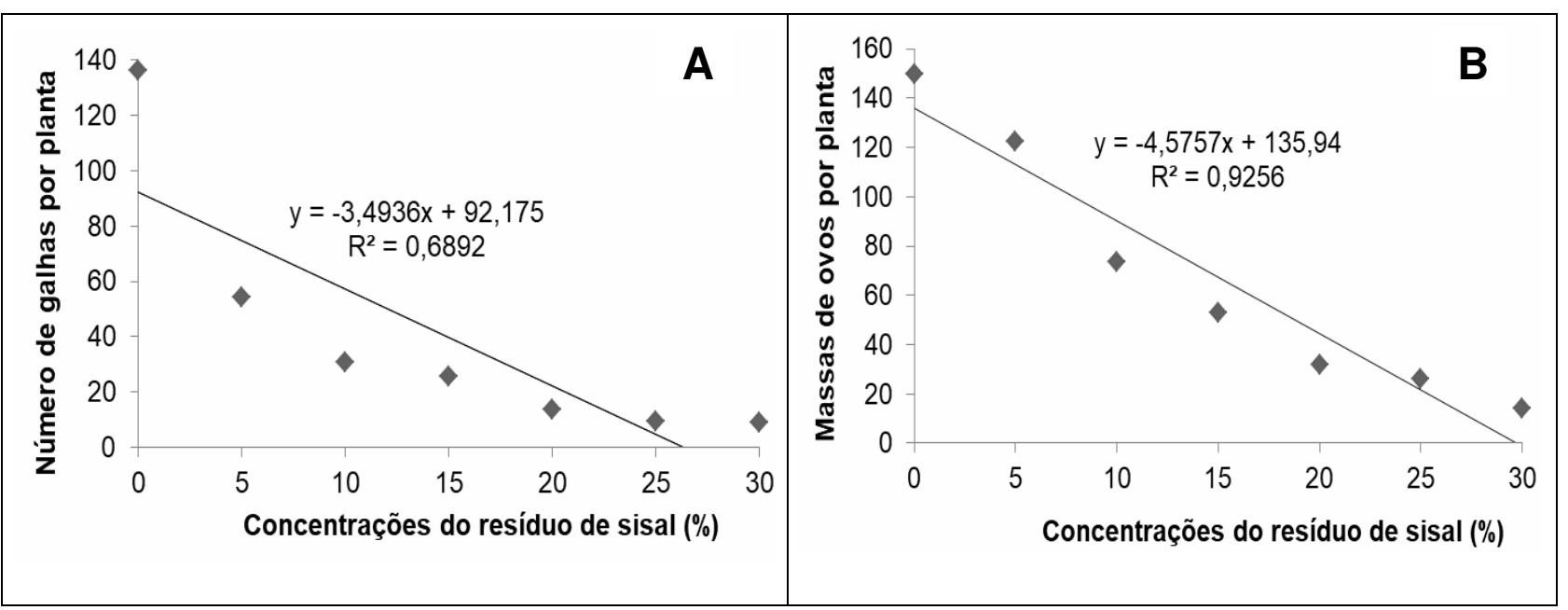

FIGURA 2. A) Número de galhas e de B) massas de ovos por planta de alface tratadas com diferentes concentrações de resíduo líquido de sisal.

Trabalho similar foi desenvolvido por Jesus et al. (2015), no qual os autores avaliaram o efeito do resíduo líquido fresco de sisal no controle de Radopholus similis na cultura da bananeira e concluíram que a concentração de $25,0 \%$ reduziu em $66,0 \%$ e $84,0 \%$ o número de juvenis no solo e nas raízes, respectivamente, quando comparado com as plantas tratadas apenas com água.

Resultados semelhantes foram encontrados para o número de galhas e de massas de ovos por grama de raiz. Em relação ao número de galhas por grama de raiz, a aplicação de concentrações crescentes do resíduo de sisal, reduziu estes danos em $70,7 \%, 71,8 \%, 72,7 \%$ e $74,2 \%$ com as concentrações de 15,0\%, 20,0\%, 25,0\% e $30,0 \%$, respectivamente, em relação ao controle (sem resíduo) (Figura 3 A).

O residuo de sisal foi eficiente na redução do número de massas de ovos por grama de raiz. A redução variou de 55,5\% (menor concentração - 5,0\%) até 92,3\% (na maior concentraçao - 30,0\%). Na concentração a 20,0\%, verificou-se apenas 1,2 massas de ovos/g de raízes, enquanto que, no tratamento controle (sem a aplicação do resíduo de sisal) observou-se 6,9 massas de ovos/g de raiz (Figura 3 B). 


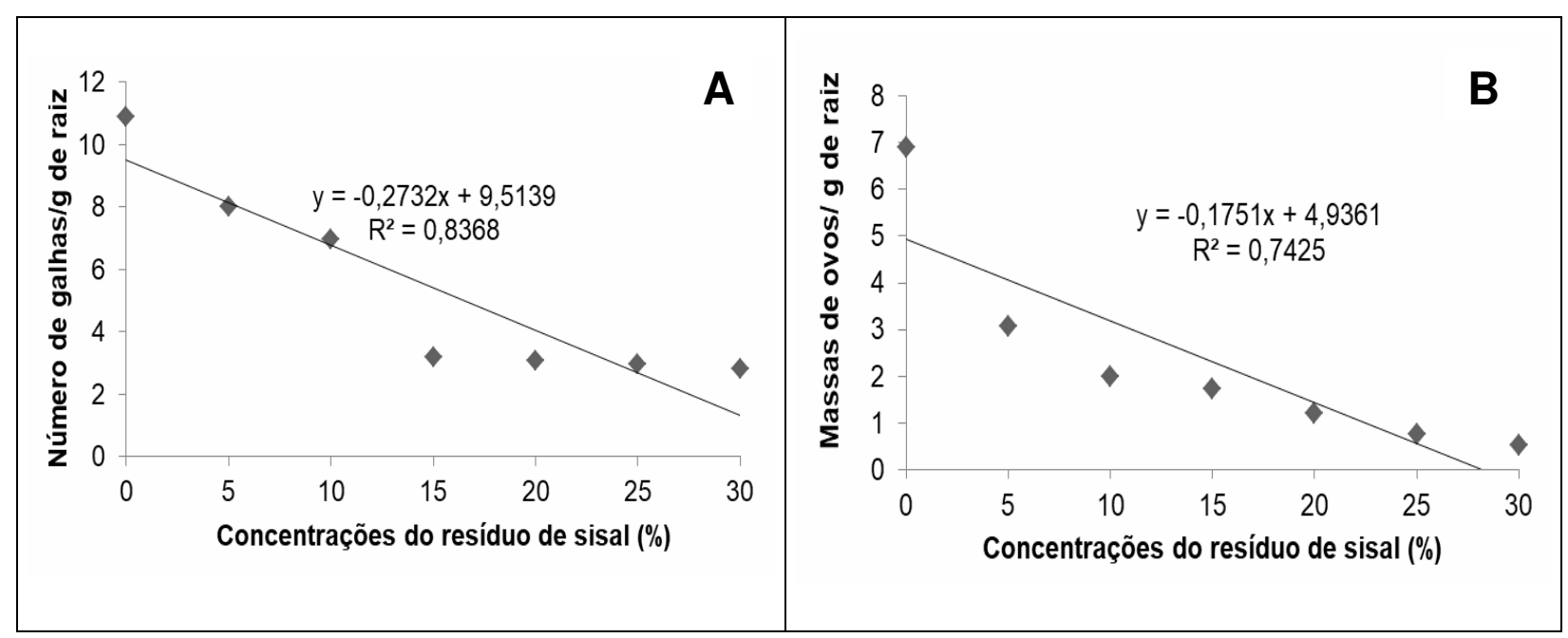

FIGURA 3. A) Número de galhas e de B) massas de ovos por planta de alface tratadas com diferentes concentrações de resíduo líquido de sisal.

Vale salientar, que nas concentrações mais elevadas $(25,0 \%$ e $30,0 \%)$ houve uma maior redução dos danos nas raízes das plantas, entretanto, estas mesmas concentrações promoveram efeito fitotóxico às plantas. Trabalho desenvolvido por Jesus et al. (2015), avaliando o efeito de diferentes concentrações (5\%, 10\%, 15\%, $20 \%$ e 25\%) do resíduo de sisal sobre Radhopolus similis em bananeira, verificaram que a aplicação do resíduo de sisal a partir da concentração de $10 \%$, promoveu efeito fitotóxico às plantas, com decréscimo linear nas massas das matérias secas da parte aérea, das raízes e do rizoma, respectivamente.

As massas de ovos presentes nas raízes são fontes de inóculo e fundamentais para a disseminação do nematoide, o que favorece a ocorrência de novos ciclos e a sobrevivência do patógeno no solo. A redução no número de massas de ovos representa uma quebra no ciclo de vida do nematoide e nos níveis populacionais do mesmo e, consequentemente, promoverá melhores condições de desenvolvimento da cultura (DAMASCENO et al., 2015).

É muito comum a presença de $M$. javanica em áreas de produção de alface, o que prejudica o sistema radicular, afetando negativamente a produtividade da cultura. $A$ descoberta de nematicidas que sejam eficientes e ambientalmente seguros é uma necessidade constante.

Costa et al. (2000) avaliaram a patogenicidade e a reprodução de $M$. incognita em tomateiros tratados com filtrados fúngicos, extratos de plantas e estercos animais. Observaram que o extrato obtido de Coffea arabica L. (cafeeiro) causou redução na biomassa da parte aérea, entretanto, reduziu o número de ovos. Segundo os autores, a mesma substância responsável pela redução do número de ovos pode ter causado toxidez à cultura do tomateiro.

Damasceno et al. (2015) aplicaram as concentrações de 0,0\%, 4,0\%, 8,0\%, $12,0 \%, 16,0 \%$ e $20,0 \%$ do resíduo líquido fresco do sisal no controle do nematóide de galhas do tomateiro e verificaram que o mesmo apresentou atividade nematicida. Segundo os autores a concentração de $20 \%$ reduziu o número de galhas e de massas 
de ovos por grama de raízes em até $73,7 \%$ e $76,0 \%$, respectivamente, em relação ao tratamento controle.

A identificação de compostos bioativos nas folhas de $A$. sisalana poderá aumentar o interesse pelo aproveitamento do seu resíduo, principalmente, para o controle de patógenos. O efeito nematicida verificado no presente estudo pode estar associada a ação de metabólitos secundários presentes no resíduo do sisal tais como, alcalóides, saponinas, terpenos, compostos fenólicos, glicosídeos, relacionadas às atividades biológicas (BOTURA et al., 2013; RIBEIRO et al., 2013; WANG et al., 2014).

Yang et al. (2015) avaliando a torta de sementes de Camellia no controle de $M$. javanica em bananeira,verificaram que as saponinas presentes na torta, apresentaram efeito nematicida sobre esses nematoides. De acordo com os autores, a torta de sementes de Camellia promoveu controle dos nematoides em condições in vitro e nas plantas de bananeira, sendo estes resultados atribuídos à presença de compostos nematicidas, incluindo saponinas e compostos voláteis.

\section{CONCLUSÕES}

Todas as concentrações de resíduo líquido de sisal fresco aplicados no solo controlaram a população de $M$. javanica na cultura da alface em condições de casa de vegetação, contudo, a concentração de $20 \%$ demonstrou efeito nematicida, além de favorecer a produção de massa seca da planta; Concentrações acima de $20 \%$ dos resíduos líquidos de sisal fresco aplicados no solo, causaram fitotoxidez em plantas de alface em condições de casa de vegetação; Trabalhos em condições de campo devem ser realizados para concentrações inferiores à $20 \%$ de resíduo líquido de sisal fresco, buscando comprovar sua eficiência sobre $M$. javanica, bem como o comportamento e interação com a microbiota do solo.

\section{REFERÊNCIAS}

BARRÊTO, A.F.; ARAÚJO, E.; BONIFÁCIO, B.F. Eficiência de extratos de Agave sisalana (Perrine) sobre o ácaro rajado Tetranychus urticae (Koch) e ocorrência de fitotoxidez em plantas de algodoeiro (Gossypium hirsutum L. r latifolium Hutch). Revista Brasileira de Agroecologia, v. 5, p. 207-215, 2010. Disponível em: http://www.scielo.br/scielo.php?script=sci_nlinks\&ref=000080\&pid=S1806-

$6690201300040001200003 \&$ Ing=pt. Acesso em 15 de mai. 2017

BONETTI, J.I.S.; FERRAZ, S. Modificações do método de Hussey \& Barker para extração de ovos de Meloidogyne exigua, em raízes de cafeeiro. Fitopatologia Brasileira, $\quad$ v. $6, \quad$ p. 533, 1981. Disponível em: http://www.scielo.br/scielo.php?script=sci_nlinks\&ref=000081\&pid=S0100204X200600120001000002\&Ing=pt.

BOTURA, M.B.; SANTOS, J.D.G.; SILVA, G.D.; LIMA, H.G.; OLIVEIRA, J.V.A.; ALMEIDA, M.A.O.; BATATINHA, M.J.M.; BRANCO, A. In vitro ovicidal and larvicidal activity of Agave sisalana Perr. (sisal) on gastrointestinal nematodes of goats. Veterinary Parasitology, 2013; v. 192, p. 211-217. Disponível em: http://dx.doi.org/10.1016/j.vetpar.2012.10.012. Acesso em 15 de jul. 2017 
CHITWOOD, D. J. Phytochemical based strategies for nematode control. Annual Review of Phytopathology, v. 40, p. 221-249, 2002. DOI:

10.1146/annurev.phyto.40.032602.130045 Acesso em: 22 de fev. 2017

COSTA, M. J. N.; CAMPOS, V.P.; PFENNING, L.H.; OLIVEIRA, D.F. Patogenicidade e reprodução de Meloidogyne incognita em tomateiro (Lycopersicon esculentum) com aplicação de filtrados fúngicos ou extratos de plantas e de estercos animais. Nematologia Brasileira, v. 24, p. 219-226, 2000.

CONAB. 2015, 27 de maio. Sisal 2015: Retrospectiva. Disponível em: http://www.conab.gov.br/OlalaCMS/uploads/arquivos/16_02_04_11_15_32_sisal_conj untura_especial_retrospectiva_2015-1.pdf

CORDEIRO, M.J.Z.; MATOS, A.P.; KIMATI, H. Doenças da bananeira. In: KIMATI H.; AMORIM, L.; REZENDE, J.A.M.; BERGAMIN FILHO, A.; CAMARGO, L.E.A. (eds.). Manual de Fitopatologia: Doenças das Plantas Cultivadas. São Paulo: Agronômica Ceres, p.99-117, 2005.

DAMASCENO, J.C.A.; SOARES, A.C.F; JESUS, F.N.; CASTRO, J.M.C. Root-knot nematode staining with artificial food dyes. Nematoda, v 3, p. 1-5, 2016. Disponível em: http://dx.doi.org/10.4322/nematoda.01816 Acesso em: 15 fev. 2017

DAMASCENO, J.C.A.; SOARES, A.C.F.; JESUS, F.N.; SANT'ANA, R.S. Sisal leaf decortication liquid residue for controlling Meloidogyne javanica in tomato plants. Horticultura Brasileira, v. 33, p. 155-162, 2015. Disponível em: http://www.scielo.br/scielo.php?script=sci_arttext\&pid=S0102-05362015000200004. http://dx.doi.org/10.1590/S0102-053620150000200004. Acesso em: 25 de jun. 2017

DOMINGUES, L. F.; BOTURA, M. B.; CRUZ, A. C. F. G.; YUKI, C. C.; SILVA, G. D.; COSTA, M. S.; et al., ; Evaluation of anthelmintic activity of liquid waste of Agave sisalana (sisal) in goats. Revista Brasileira de Parasitologia Veterinária, v. 19, p. 270272, $2010 . \quad$ Disponível em: http://www.scielo.br/scielo.php?script=sci_arttext\&pid=S1984-29612010000400018. http://dx.doi.org/10.1590/S1984-29612010000400018. Acesso em: 16 de ago. 2017

FILHO, J.A.M.; SILVA, S.A.; FILHO, R.D.T. Degradations kinects and aging mechanisms on sisal fibers cement composite systems. Cement \& Concrect Composite, v. 40, p. 30-39, 2013. Disponível em: http://www.sciencedirect.com/science/article/pii/S0958946513000504.

https://doi.org/10.1016/j.cemconcomp.2013.04.003. Acesso em 30 de set. 2017

FREIRE, G. F.; LEITE, D. T.; PEREIRA R. A.; MELO, B. A.; SILVA, J. F.; MARACAJÁ, P. B.; Bioatividade de Solanum melongena L. E Capsicum annuum L. sobre Callosobruchus maculatus (COLEOPTERA: BRUCHIDAE). Acta biológica Colombiana. v. 21, n. 1, 2016. Disponível em: 
<http://dx.doi.org/10.15446/abc.v21n1.45775>. doi: 10.15446/abc.v21n1.45775. Acesso em: 14 de ago. 2017

HORTIBRASIL (INSTITUTO BRASILEIRO DE QUALIDADE EM HORTICULTURA). Alface em números: novidades no mercado - frutas e hortaliças frescas. [S.I.], 24 set. 2013. Disponível em:http://www.hortibrasil.org.br/. Acesso em: 15 Fev. 2017.

JESUS, F. N.; DAMASCENO, J. C. A.; BARBOSA, D. H. S. G.; MALHEIRO, R.; PEREIRA, J. A.; SOARES, A. C. F. Control of the banana burrowing nematode using sisal extract. Agronomy for Sustainable Development, v. 35, n. 2, p. 783 - 791, 2015. Disponível em: <https://doi 10.1007/s13593-014-0264-z. Acesso em 15 de set. 2017

JÚNIOR, J.R.V.; FERNANDES, C.F.; SANGI, S.C.; FREIRE, T.C.; FONSECA, A.S. Extratos de espécies de Capsicum no controle in vitro de patógenos de importância agrícola. Enciclopédia Biosfera, v.14 n.25; p. 673- 686, 2017. Disponível em: http://www.conhecer.org.br/enciclop/2017a/agrar/producao\%20de\%20alface.pdf. DOI: 10.18677/EnciBio_2017A52. Acesso em: 20 de set. 2017

JÚNIOR, J.C.M.; SENE, R.C.A.; THULER, R.T.; TORRES, J.L.R.; JÚNIOR, F.A.D. Utilização de rizobactérias no controle da lagarta-falsa medideira na cultura da soja.

Enciclopédia Biosfera, v.14 n.25; p. 1053- 1063, 2017. DOI: 10.18677/EnciBio_2017A102. Acesso em 22 de set. 2017

MAIA, T. F.; DONATO, A.; FRAGA, M. E. Atividade antifúngica de óleos essenciais de plantas. Revista Brasileira de Produtos Agroindustriais, v. 17, n. 1, p.105-116, 2015. Disponível em: <https://doi.org/10.15871/1517-8595/rbpa.v17n1p105-116>. doi: 10.15871/1517-8595/rbpa.v17n1p105-116. Acesso em 14 de ago. 2017

ORNAT, C., SORRIBAS, F.J., 2008. Integrated management of root-knot nematodes in mediterranean 1019 horticultural crops. In: CIANCO, A., MUKERJI, K.G. (Eds.), Integrated Management and Biocontrol of Vegetable and Grain Crops Nematodes. Springer, Dordrecht (NLD), (Integrated management of plant pests and diseases, vol. 2), 2008. pp. 295-319. Disponível em: : <https://doi.10.1007/978-1-4020-6063-2. Acesso em 14 de ago. 2017

RIBEIRO, H. B.; RIBEIRO, R.C.F.; XAVIER, A.A.; CAMPOS, V.P.; DIAS-ARIEIRA, C.R.; MIZOBUTSI, E.Y. Resíduos de frutos de pequi no controle do nematóide das galhas em tomateiro. Horticultura Brasileira, v. 30, p. 453-458, 2012. Disponivel em: http://dx.doi.org/10.1590/S0102-05362012000300016. Acesso em: 05 de ago. 2017

RIBEIRO, B. D.; ALVIANO, D. S.; BARRETO, D. W.; COELHO, M. A. Z.Functional properties of saponins from sisal (Agave sisalana) and juá (Ziziphus joazeiro): Critical micellar concentration, antioxidant and antimicrobiala ctivities. Colloids and Surfaces A: Physicochemical and Engineering Aspects, v.436, p. 736-743, 2013. Disponível em: https://doi.org/10.1016/j.colsurfa.2013.08.007. Acesso em: 29 de jul. 2017 
RIBEIRO, B. D.; COELHO, M. A. Z.; MARRUCHO, I.M. Extraction of saponins from sisal (Agave sisalana) and juá (Ziziphus joazeiro) with cholinium-based ionic liquids and deep eutectic solvents .European Food Researchand Technology, v.237, p. 965-975, 2013. Disponível em: https://dx.doi.org/10.1007/s00217-013-3068-9. Acesso em 27 de set. 2017

SALA, F. C.; COSTA, C. P da. Retrospectiva e tendência da alfacicultura brasileira. Horticultura Brasileira, p. 187-194, 2012. Disponível em: http://www.scielo.br/scielo.php?script=sci_arttext\&pid=S0102. Acesso em 20 de set. 2017.

SILVA, R.G.; SANTOS, T.F.S.; SILVA, I.C.D.; SILVA, M.B.S.; BORGES, G.A.N. Reaçao de genótipos de soja ao nematóide das lesoes radiculares. Enciclopédia Biosfera, v.11 n.22; p. 187-193, 2015. Disponível em: http://www.conhecer.org.br/enciclop/2015c/agrarias/Reacao\%20de\%20genotipos.pdf. DOI: http://dx.doi.org/10.18677/Enciclopedia_Biosfera_2015_219

SUINAGA, F. A.; SILVA, O. R. R. F.; COUTINHO, W. M. Cultivo de sisal na região Semi-árida do nordeste brasileiro. Campina Grande: EMBRAPA Algodão, 2006. 42p.

WANG, Y.; LI, X.; SUN, H.; YI, K.; ZHENG, J.; HAO, Z. Biotransformation of steroidal saponins in sisal (Agave sisalana Perrine) to tigogenin by a newly isolated strain from a karst area of Guilin, Chin. Biotechnology \& Biotechnological Equipment, v. 28, n. 6, p. 1024-1033, 2014. Disponível em: http://dx.doi.org/10.1080/13102818.2014.978199. Acesso em 27 de set. 2017

WILCKEN, S.R.S.; GARCIA, M.J.M.; SILVA, N. Resistência de alface do tipo americana a Meloidogyne incognita raça 2. Nematologia Brasileira, v. 29, p. 267-271, 2005. Acesso em: 18 de ago. 2017

YANG, X.; WANG, X.; WANG, K.; SU, L.; LI, H.; LI, R.; SHEN, Q. The nematicidal effect of Camellia seed cake on root-knot nematode Meloidogyne javanica of banana. Plos One, v. 10, p. 1-18, 2015. Disponível em: https://doi.org/10.1371/journal.pone.0119700. Acesso em: 18 de ago. 2017 